\title{
Perfusión y función ventricular en SPECT cardiaco y coronariografía del mismo día según localización en enfermedad de un vaso
}

\author{
PATRICIO GONZÁLEZ ${ }^{1}$, GASTÓN DUSSAILLANT², DANIELA GUTIÉRREZ \\ ISABEL BERROCAL ${ }^{1}$, RITA ALAY ${ }^{1, a}$, SONIA OTÁROLA ${ }^{1, a}$
}

'Sección Medicina Nuclear, Departamento de Medicina. ${ }^{2}$ Departamento Cardiovascular, Hospital Clínico Universidad de Chile. ${ }^{\mathrm{a} T M}$.

Proyecto Desarrollo de Líneas Prioritarias de Investigación, Financiado OAIC 053/04, Hospital Clínico Universidad de Chile.

Recibido el 4 de junio de 2012, aceptado el 25 de junio de 2013.

Correspondencia a: Dr. Patricio González Espinoza Hospital Clínico Universidad de Chile, Departamento de Medicina, Sección Medicina Nuclear

Santos Dumont 999-1 p, E-156, Independencia, Santiago, Chile.

Teléfono: 562 - 777-0569

Fax: 562 - 777-7618

E-mail: pgonzalez@hcuch.cl

\section{Single-photon emission computed tomography for the assessment of ventricular perfusion and function}

\begin{abstract}
Background: Single-photon emission computed tomography (SPECT) can be used as a non-invasive tool for the assessment of coronary perfusion. Aim: To assess ventricular perfusion and function by SPECT in patients with single vessel coronary artery disease. Material and Methods: Among patients with indications for a coronary artery angiography, those with significant lesions in one vessel, were selected for the study. Within 24 hours, cardiac SPECT examinations on basal conditions and after high doses of dipyridamole, were performed. SPECT data from 38 patients with a low probability of coronary artery disease was used for comparisons. Results: Ten patients aged $61 \pm 8$ years (seven men) were studied. Visual analysis of SPECT revealed signs suggestive of ischemia in eight patients. The remaining two patients did not have perfusion disturbances. SPECT detected eight of ten abnormal vessels reported in the coronary artery angiography. There were two false negative results Summed stress, summed rest and summed difference scores were $9.78 \pm 6.51,3.22$ \pm 5.07 and $6.33 \pm 4.97$, respectively. The ejection fractions under stress and at rest were $53 \pm 11.7 \%$ and $61 \pm 15.7 \%$ respectively $(p<0.01)$. The figures for the control group were $69.1 \pm 13.5 \%$ and $75.2 \pm 12.04 \%$ respectively (significantly different from patients). Two patients had a summed motion score above 14.9. Likewise, two patients had a summed thickening score above 10.9. Conclusions: SPECT detected $80 \%$ of coronary lesions found during coronary artery angiography. Visual analysis of perfusion is highly reliable for diagnosis. Quantitative parameters must be considered only as reference parameters.
\end{abstract}

(Rev Med Chile 2013; 141: 1136-1142).

Key words: Coronary vessels; Tomography, emission-computed; single-photon; Ventricular function.
L a tomografía computarizada por emisión de un solo fotón (SPECT) cardiaco sincronizado a electrocardiograma (ECG) es un método rutinario en el diagnóstico y evaluación de la enfermedad coronaria (EC). Su sensibilidad y especificidad se encuentran entre $70 \%$ y $90 \% 0^{1,2}$.

No hay estudios comparando el SPECT con compuestos tecneciados y coronariografía realizados el mismo día y que valore el rendimiento en lesiones proximales y distales en sujetos con enfermedad de 1 vaso, analizando también en qué medida parámetros de perfusión y de función ventricular contribuyen en la detección de la $\mathrm{EC}^{3}$.

El objetivo ha sido comparar el rendimiento de 
la perfusión y función ventricular en enfermedad coronaria de un vaso, según localización y severidad de la estenosis en angiografía coronaria del mismo día.

\section{Material y Método}

Estudio prospectivo en 10 pacientes, edad promedio \pm sd de $61 \pm 8$ años, incluyendo 7 hombres y 3 mujeres (Tabla 1). Criterios de inclusión, paciente con angor (angina estable o inestable), alta probabilidad de enfermedad coronaria y que requiere coronariografía. La insuficiencia cardiaca o la insuficiencia mitral no fueron contraindicaciones para realizar el cintigrama miocárdico. Se excluyeron los que habían tenido un infarto. El infarto previo se descartó en base a los antecedentes, ECG en todos los pacientes (ausencia de onda Q patológica) y con ecocardiograma cuando estuvo disponible (ausencia de trastorno de motilidad y cicatriz). También se excluyeron enfermos previamente revascularizados.

Se realizó en primer lugar como técnica de elección la coronariografía. Se seleccionaron los pacientes con lesiones coronarias significativas de un vaso. Se ha utilizado como criterio para lesión crítica una estenosis $\geq 70 \%$. Por otro lado, se ha considerado estrechez no significativa un valor $\leq 50 \%$. En la coronariografía, interpretada por un operador (GDN), ciego a la información del SPECT, se realiza estimación visual del grado de estenosis. Dentro de $24 \mathrm{~h}$ fueron evaluados con SPECT cardiaco. Se analiza resultados por paciente $(n=10)$ y vaso coronario $(n=30)$, lo que agrega una compensación estadística a la muestra pequeña de sólo 10 pacientes. La revascularización fue realizada luego de completado el estudio con SPECT y de acuerdo con la indicación de los médicos tratantes, ya que la condición clínica lo hizo posible en todos los casos. Tratándose de enfermedad de un vaso el tratamiento fue la angioplastía.

Se compara además a SPECT cardiaco en grupo control que comprende 38 pacientes de ambos sexos, con baja probabilidad de enfermedad coronaria y SPECT con alta dosis de dipiridamol reportados en forma independiente, como normales. El SPECT cardiaco se efectúa en protocolo de uno (estrés en el primer día en los 10 pacientes) y dos días (fase de reposo el segundo día en 2 casos), con dipiridamol en alta dosis $(0,852 \mathrm{mg} / \mathrm{kg})$, con $\mathrm{Tc}^{99 \mathrm{~m}}$ Sestamibi, con técnica sincronizada a ECG

\section{Tabla 1. Datos clínicos generales} en los 10 pacientes

\begin{tabular}{|cccl|}
\hline Caso & Sexo & Edad & Diagnóstico clínico \\
\hline 1 & M & 56 & Angina estable \\
\hline 2 & M & 53 & Angina estable \\
\hline 3 & M & 69 & $\begin{array}{l}\text { Insuficiencia cardiaca CF III. } \\
\text { Insuficiencia mMitral }\end{array}$ \\
\hline 4 & M & 51 & Angina estable \\
\hline 5 & F & 76 & Angina inestable \\
\hline 6 & M & 68 & Angina estable \\
\hline 7 & M & 53 & Angina estable \\
\hline 8 & M & 59 & Angina inestable \\
\hline 9 & F & 61 & Angina estable \\
\hline 10 & F & 63 & Angina estable \\
\hline
\end{tabular}

en ambas fases, salvo un paciente con inadecuada señal electrocardiográfica.

La perfusión bajo estrés y reposo se interpreta visualmente por 2 observadores en forma ciega, evaluando presencia de segmentos normales, isquémicos y necróticos, incluyendo valoración porcentual semicuantitativa para extensión y severidad $^{4}$.

Adicionalmente, mediante parámetros cuantitativos del mapa polar comparando con base de datos normal, SSS (score sumado de estrés) que se relaciona con defecto de perfusión en estrés, SRS (score sumado de reposo), defecto de perfusión persistente en reposo y SDS (score sumado de diferencias), que se relaciona al área reversible al comparar defecto de estrés y reposo.

La función ventricular se determina considerando fracción de eyección (FE), de estrés y reposo. Del mismo modo, con SMS (score de motilidad en estrés), RMS (score de motilidad en reposo), STS (score de engrosamiento en estrés) y RTS (score de engrosamiento en reposo), que se relacionan a la contractilidad y engrosamientos segmentarios en todo el ventrículo izquierdo, respectivamente.

Tanto la perfusión como función ventricular se correlacionan a lo reportado en los vasos coronarios, respecto a severidad y localización de la estenosis. Para el análisis estadístico se utiliza la prueba de Student en aquellos con distribución normal (FE, SRS y SDS) y la prueba de Wilcoxon Mann Whitney en los restantes, definidos mediante el test de D'Agostino 5 . 
Fue aprobado por el Comité de Ética del Hospital Clínico de la Universidad de Chile, Proyecto OAIC 053/04. Todos los pacientes firmaron consentimiento informado.

\section{Resultados}

En la Tabla 2, se detalla los hallazgos de la angiografía coronaria para los 10 pacientes. Enfermedad de 1 vaso con estenosis significativa, 5 en descendente anterior (ADA), 3 en coronaria derecha $(C D)$ y 2 en circunfleja (CX). Hubo 3 lesiones proximales, 2 en segmento medio y 5 distales. En los 2 pacientes con lesiones oclusivas (100\%), casos 1 y 7 se demostró presencia significativa de colaterales, no evidenciando infarto. El ECG con alta dosis de dipiridamol resultó normal en 6 casos, en 3 demostró isquemia y en el otro fue positivo por respuesta clínica y dolor torácico.

\section{Parámetros visuales de perfusión}

\section{Análisis por paciente y vaso coronario}

La perfusión en el análisis visual por paciente reveló que 8 de los 10 casos estudiados presentaron en el SPECT cardiaco defectos sugerentes de isquemia (casos 1 a 4 y 6 a 9) y en 2 de estos 8 , se detectó además área de necrosis en el mismo territorio (casos 3 y 9), por lo tanto, sensibilidad de $80 \%$. Los $2 / 10$ pacientes restantes no presentaron alteraciones de la perfusión (20\% de falsos negativos, casos 5 y 10).
En la Tabla 3 se muestra los porcentajes de estenosis de las arterias coronarias, su localización y la extensión de los defectos de perfusión en el SPECT.

De los 30 vasos coronarios, la coronariografía estuvo alterada en 10, de los cuales 8 fueron detectados por el SPECT (Figura 1). Por otro lado, 20 arterias coronarias fueron normales y en ninguna de ellas se encontró alteración de perfusión. Por

Tabla 2. Hallazgos en la coronariografía en los 10 pacientes y $\mathbf{3 0}$ territorios coronarios, detallando localización, grado de estenosis, según vaso

\begin{tabular}{|c|c|c|c|c|c|c|}
\hline Caso & $\begin{array}{c}\text { ADA } \\
(\%)\end{array}$ & $\mathbf{L}$ & $\begin{array}{l}\text { CD } \\
(\%)\end{array}$ & $\mathbf{L}$ & $\begin{array}{l}\text { CX } \\
(\%)\end{array}$ & $\mathbf{L}$ \\
\hline 1 & 0 & NA & 100 & P & 50 & $P$ \\
\hline 2 & 88 & P & 0 & NA & 0 & NA \\
\hline 3 & 50 & $P$ & 70 & D & 40 & $P$ \\
\hline 4 & 90 & $\mathrm{M}$ & 0 & NA & 0 & NA \\
\hline 5 & 70 & $D$ & 40 & D & 50 & $P$ \\
\hline 6 & 0 & NA & 70 & D & 0 & NA \\
\hline 7 & 100 & D & 0 & NA & 50 & $M$ \\
\hline 8 & 90 & P & 20 & $P$ & 0 & NA \\
\hline 9 & 30 & $\mathrm{M}$ & 0 & NA & 80 & $D$ \\
\hline 10 & 0 & NA & 0 & NA & 90 & $M$ \\
\hline
\end{tabular}

$\mathrm{L}=$ Localización; $\mathrm{ADA}=$ Arteria Descendente Anterior; $\mathrm{CD}=$ Coronaria Derecha; $\mathrm{CX}=$ Circumfleja; $\mathrm{NA}=$ No Aplica; $\mathrm{P}=$ Proximal; $\mathrm{M}=$ Media; $\mathrm{D}=$ Distal.

Tabla 3. Comparación de SPECT y vaso coronario

\begin{tabular}{|c|c|c|c|c|c|c|c|c|}
\hline \multicolumn{2}{|c|}{ Angiografía } & \multirow{2}{*}{$\begin{array}{l}\text { SPECT } \\
\text { DF (\%) }\end{array}$} & \multicolumn{2}{|c|}{ Angiografía } & \multirow{2}{*}{$\begin{array}{l}\text { SPECT } \\
\text { DF (\%) }\end{array}$} & \multicolumn{2}{|c|}{ Angiografía } & \multirow{2}{*}{$\begin{array}{l}\text { SPECT } \\
\text { DF ( } \%\end{array}$} \\
\hline ADA (\%) & $\mathbf{L}$ & & CD (\%) & $\mathbf{L}$ & & CX (\%) & $\mathbf{L}$ & \\
\hline 0 & NA & 0 & 100 & P & 12 & 50 & $P$ & 0 \\
\hline 88 & P & 22 & 0 & NA & 0 & 0 & NA & 0 \\
\hline 50 & $\mathrm{P}$ & 0 & 70 & D & 24 & 40 & $P$ & 0 \\
\hline 90 & M & 18 & 0 & NA & 0 & 0 & NA & 0 \\
\hline 70 & D & 0 & 40 & D & 0 & 50 & $P$ & 0 \\
\hline 0 & NA & 0 & 70 & D & 6 & 0 & NA & 0 \\
\hline 100 & D & 11 & 0 & NA & 0 & 50 & M & 0 \\
\hline 90 & $P$ & 22 & 20 & $P$ & 0 & 0 & NA & 0 \\
\hline 30 & M & 0 & 0 & NA & 0 & 80 & D & 30 \\
\hline 0 & NA & 0 & 0 & NA & 0 & 90 & M & 0 \\
\hline
\end{tabular}

$\mathrm{ADA}=$ Arteria Descendente Anterior; $\mathrm{CD}=$ Coronaria Derecha; $C X=$ Circumfleja; $\mathrm{L}=$ Localización; $\mathrm{DF}=$ Defecto; $N A=$ No Aplica; $\mathrm{P}=$ Proximal; $\mathrm{M}=$ Media; $\mathrm{D}=$ Distal. 

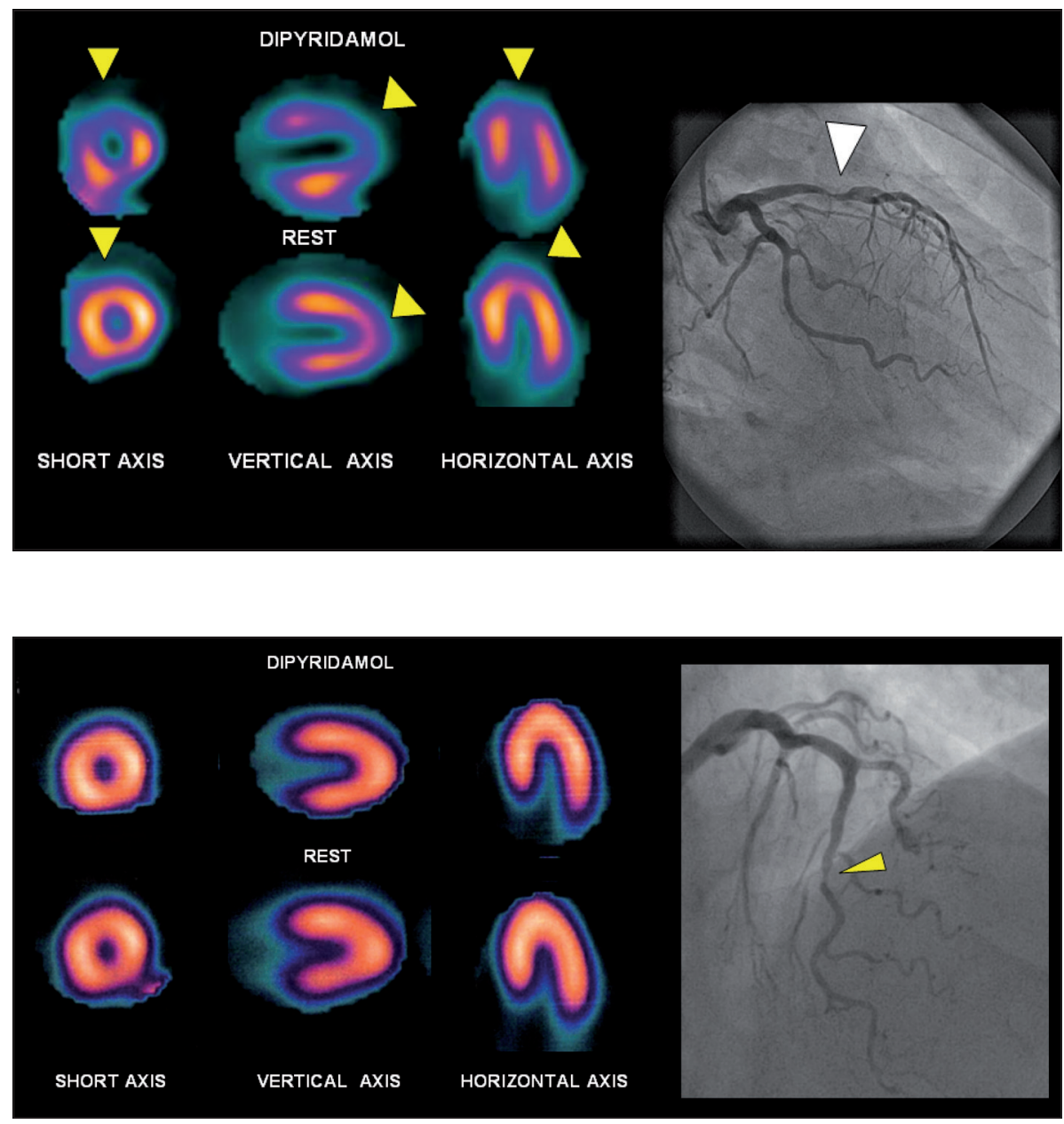

Figura 1. Caso 2, Lesión proximal $88 \%$ en ADA (Flecha blanca a derecha), SPECT muestra extensa isquemia anteroseptoapical, con defecto en fase de dipiridamol que revierte en reposo (Flechas amarillas a izquierda).

Figura 2. Caso 5, Lesión distal $70 \%$ en ADA (Flecha amarilla a derecha), SPECT normal con distribución homogénea en los 3 ejes).

ende, la sensibilidad fue de $80 \%$ y la especificidad de $100 \%$. Los 2 falsos negativos correspondieron a pacientes mujeres, una de ellas con lesión de $70 \%$ en el segmento distal de la coronaria descendente anterior (Figura 2) y la otra con estenosis de circunfleja de $90 \%$ en el segmento medio. Todas las lesiones proximales fueron detectadas independiente del vaso coronario en que estaban localizadas. Hubo 3 lesiones proximales en ADA, 2 en $\mathrm{CD}$ y 3 en CX.

\section{Parámetros cuantitativos de perfusión Análisis por paciente}

El SSS fue de 9,78 \pm 6,51 en todo el grupo y aunque es mayor, comparado al grupo control de $6,61 \pm 5,64$, no es estadísticamente diferente $(\mathrm{p}=$ 0,148 ) y sólo $1 / 9$ tuvo cifra superior a 18 (límite de
2 DS). El SRS fue de 3,22 $\pm 5,07$ en todo el grupo que comparado al grupo control de 3,45 $\pm 3,49$ no es estadísticamente diferente $(p=0,902)$ y en $2 / 9$ casos fue superior a 10,4 (límite de 2 DS).

El SDS fue de 6,33 $\pm 4,97$ en todo el grupo que comparado al grupo control de 3,03 $\pm 3,66$ no es estadísticamente diferente (NS, $p=0,089$ ) y en $2 / 9$ fue superior a 10,34 (límite de 2 DS).

\section{Parámetros cuantitativos de función Análisis por paciente}

Respecto a parámetros de función ventricular, la fracción de eyección posterior a estrés fue de $53,4 \% \pm 11,7$ y en reposo de $61,3 \% \pm 15,7$ $(\mathrm{p}<0,008)$. Respecto a grupo control, de 69,1\% $\pm 13,5$ y $75,2 \% \pm 12,04$ ( $\mathrm{p}=0,001$ entre estos), la diferencia es estadísticamente significativa para 
cada uno de ellos $(p=0,003$ y $p=0,032)$, respectivamente. Sólo 2 pacientes bajo estrés y reposo, presentan valores por debajo de $42 \%$ y $51 \%$ (límite de 2 DS).

El SMS fue de $8,78 \pm 8,56$ y el RMS de 6,78 $\pm 13,2(\mathrm{p}=0,185)$. Respecto a grupo control, de $4,18 \pm 5,37$ y $1,87 \pm 3,71$ ( $p=0,029$ entre estos), la diferencia no es estadísticamente significativa ( $\mathrm{p}=0,089$ y $\mathrm{p}=0,256)$, respectivamente. Sólo 2 pacientes presentan valores por encima de $14,9 \mathrm{y}$ 9,3 (límite de 2 DS) del grupo control.

El STS fue de 6,22 $\pm 8,73$ y el RTS de $4 \pm 9,38$ $(\mathrm{p}=0,216)$. Respecto a grupo control, de 2,66 $\pm 4,13$ y $0,58 \pm 2,15$ ( $\mathrm{p}=0,004$ entre estos), la diferencia no es estadísticamente significativa $(\mathrm{p}=0,251$ y $\mathrm{p}=0,617)$, respectivamente. Sólo 2 pacientes bajo estrés y 1 en reposo presentan valores por encima de 10,9 y 4,88 (límite de 2 DS) del grupo control, respectivamente.

\section{Discusión}

Las enfermedades cardiovasculares son la primera causa de mortalidad en los países desarrollados. Una de sus etiologías específicas corresponde a la enfermedad coronaria que se manifiesta de diversa forma tanto en procesos agudos como crónicos $^{6,7}$.

Los procedimientos de imágenes juegan hoy en día un rol importante en la detección de la EC. Entre los más utilizados cabe destacar, la ecocardiografía, SPECT cardiaco, angiotac, resonancia magnética y angiografía coronaria ${ }^{8,9}$.

Las técnicas de cardiología nuclear se emplean rutinariamente en el diagnóstico y evaluación de EC. En especial el SPECT cardiaco con compuestos tecneciados y con técnica de ECG sincronizada que posibilita obtener información de la perfusión, así como de la función ventricular ${ }^{1}$.

Se ha reportado sensibilidad y especificidad de alrededor de $90 \%{ }^{1,2}$.

Sin embargo, en algunas circunstancias su rendimiento puede ser menor, por razones técnicas o según severidad y forma de presentación de la enfermedad coronaria.

Una publicación específica que evalúe el rendimiento de la técnica según la localización de las lesiones no ha sido reportada para Tc99m-Sestamibi con técnica sincronizada a ECG y que utilice perfusión y función ventricular simultáneamente y además con comparación a coronariografía del mismo día.

Esto último conlleva un esfuerzo importante en realizar los procedimientos aunque con el beneficio de otorgar valiosa información al no existir diferencia de tiempo en la práctica, entre ambos procedimientos de tal forma que la comparación resulta mucho más certera.

Todos estos elementos son el fundamento que motiva esta publicación agregando además que ha sido realizada en enfermedad de 1 vaso coronario.

A este respecto es interesante destacar el trabajo de De Pasquale EE et $\mathrm{al}^{10}$, quienes vieron que la sensibilidad para la detección de compromiso de un vaso coronario, sólo con el bullseye fue $78 \%$ para la $\mathrm{ADA}, 89 \%$ para la arteria coronaria derecha y $65 \%$ para la circunfleja.

El compromiso de enfermedad coronaria de sólo un vaso puede ser predicho con bastante exactitud en forma no invasiva mediante análisis segmentario de las imágenes con talio ${ }^{11}$.

Ambos trabajos sustentan que el modelo de EC de un vaso resulta adecuado para valorar en forma más pura el análisis de las características del SPECT miocárdico ${ }^{12,13}$.

En primer lugar, los resultados obtenidos muestran una interesante concordancia entre la coronariografía y el SPECT miocárdico, a pesar de ser técnicas basadas en aspectos diferentes como son la estructura y función del VI, es decir, anatomía del árbol coronario y flujo miocárdico.

En segundo término y en relación con la localización de las lesiones coronarias, destaca que las proximales son evidenciadas en todos los casos, explicable por la mayor masa miocárdica en riesgo que hace más fácil su detección respecto a lesiones medias o distales ${ }^{12,14}$.

En este sentido hay concordancia con el trabajo de Pichard $\mathrm{AD}$ et $\mathrm{al}^{15}$, que mostraron que una perfusión normal en el septum, que forma parte del territorio irrigado por la $\mathrm{ADA}$, prácticamente excluye la presencia de estenosis proximal significativa en este vaso coronario, debido a que la masa miocárdica septal es mayor.

Hay otros trabajos que han relacionado la localización de las lesiones coronarias con el SPECT como el de Joon-Kee Yoon et $\mathrm{al}^{16}$, que intentan predecir estenosis proximal de la arteria descendente anterior, basándose en los patrones de los defectos de perfusión evidenciados en el cintigrama con $\mathrm{Tl}^{201}$, encontrando una sensibilidad de $72 \%$, una 
especificidad de $95 \%$ y una exactitud de $91 \%$. Estos patrones del mapa polar tenían la mayor extensión ya que incluían el territorio septal.

En otro aspecto, Verbernea $\mathrm{HJ}$ et a $\mathrm{a}^{17}$ evaluaron el SPECT con $201 \mathrm{Tl}$, concluyendo que la presencia de redistribución en el ventrículo derecho junto a alteración en el ventrículo izquierdo, era indicador de compromiso proximal de la coronaria derecha. Este hallazgo también se correlaciona con mayor masa miocárdica del territorio en riesgo.

En tercer término es importante enfatizar que la interpretación visual aparece con mejor rendimiento en la detección que los parámetros puramente cuantitativos, explicable por la dispersión de estos últimos. Esto es concordante con la impresión aceptada que el análisis visual por un observador entrenado es fundamental para detectar con adecuada sensibilidad y especificidad la enfermedad coronaria. En varios trabajos además se reporta el uso de interpretación visual por 2 ó 3 observadores independientes como referente equivalente a un patrón de oro ${ }^{9,18,19}$. Los parámetros cuantitativos aunque han mejorado significativamente, son elementos de apoyo y sólo deben ser empleados como auxiliares a la interpretación visual para evaluación y diagnóstico. Por la dispersión de datos, amplios, tienen menor sensibilidad.

Aquellos de perfusión, si bien son derivados de imágenes al momento del máximo estrés, son afectados por condiciones como hipertrofia y trastornos de conducción (BCRI). Los de función ventricular son obtenidos en la etapa posterior al estrés y además pueden ser afectados por alteraciones regionales de perfusión, lo que limita evaluar los trastornos de motilidad segmentaria así como el engrosamiento.

Estos resultados son concordantes a la impresión que en la práctica clínica lo más útil es el SPECT de perfusión analizado visualmente en sus fases de estrés y reposo y en menor grado la motilidad y parámetros cuantitativos.

Limitaciones, grupo pequeño, aunque se compensa con el análisis en los 30 territorios coronarios, compara coronariografía con SPECT. Los parámetros cuantitativos no se evaluaron regionalmente, por el grupo reducido de casos con EC. Sin embargo, por tratarse de lesiones de 1 vaso coronario, las alteraciones encontradas son probablemente correspondientes al territorio de la coronaria afectada.
Ventajas, mismo día, práctica clínica, un vaso, el análisis por vaso ha permitido evaluar tanto la sensibilidad como especificidad. Grupo control de dipiridamol. Se ha empleado alta dosis de dipiridamol.

\section{Conclusiones}

En esta serie reducida de pacientes con estudio en $24 \mathrm{~h}$, comparables a series con mayor número de casos, el SPECT cardiaco y la coronariografía se correlacionan muy significativamente, no obstante que se trata de dos métodos que evalúan aspectos diferentes del corazón, uno el flujo coronario y el otro la anatomía de las arterias coronarias.

Todas las lesiones coronarias proximales son detectadas independiente del vaso explicable por inducción de isquemia en proporción a masa miocárdica en riesgo de importante extensión.

Los falsos negativos corresponden a mujeres con lesiones significativas en tercio medio o distal de descendente anterior o circunfleja con esperable menor territorio miocárdico amenazado.

Por ende, no sólo es clave el grado de estenosis, sino también la localización de las lesiones, cuando se analiza los factores que inciden en la detectabilidad.

El análisis visual de la perfusión es suficiente para un diagnóstico certero en la práctica clínica $\mathrm{y}$ en el paciente individual.

Por su dispersión, los parámetros cuantitativos de perfusión y función, pueden servir en grupos de sujetos, pero son de apoyo limitado en el paciente evaluado aisladamente.

De los parámetros cuantitativos, la fracción de eyección global aparece como el indicador con mayor valor.

Los parámetros cuantitativos deben en todo caso, ser considerados sólo de referencia y orientación y no ser sobrevalorados.

Agradecimiento: A la Srta. Amanda Muñoz Negrón por apoyo secretarial.

\section{Referencias}

1. González P, Massardo T, Muñoz A, Jofré J, Rivera A, Yovanovich $\mathrm{J}$, et al. Is the addition of ECG gating to Technetium-99m sestamibi SPET of value in the assessment of myocardial viability? An evaluation based on 
two-dimensional echocardiography following revascularization. Europ J Nucl Med 1996; 23: 1315-22.

2. Massardo T, González P, Humeres P, Chamorro H, Ayala F, Yovanovich J, et al. Simultaneous assessment of function and perfusion dipyridamole-hangrip Tc99m sestamibi imaging in chronic coronary artery disease. Annals Nucl Med 1999; 13: 121-5.

3. Azzarelli S, Galassi AR, Foti R, Mammana C, Musumeci S, Giuffrida G, et al. Accuracy of 99m Tc-tetrofosmin myocardial tomography in the evaluation of coronary artery disease. J Nucl Cardiol 1999; 6 (2): 183-9.

4. González P, Massardo T, Coll C, Humeres P, Sierralta P, Jofré MJ, Et al. The predictive value of $201 \mathrm{Tl}$ restredistribution and $18 \mathrm{~F}$-fluorodeoxyglucose SPECT for wall motion recovery after recent reperfused myocardial infarction. Ann Nucl Med 2004 Apr; 18 (2): 97-103.

5. Berrocal I, Padilla P, González P. (12 de abril de 2012). Estudio comparativo de SPECT gatillado, analizando parámetros cuantitativos con reorientación convencional y en posición anatómica sin reorientación. Alasbimn Journal, ISSN: 0717-4055. URL: http://www.alasbimnjournal.net/a/64.

6. Beller GA and Heede RC. SPECT Imaging for Detecting Coronary Artery Disease and Determining Prognosis by Noninvasive Assessment of Myocardial Perfusion and Myocardial Viability. J. Cardiovasc. Trans. Res. 2011; 4: 416-24.

7. Yoshinaga K, Manabe O, Tamaki N. Physiological Assessment of Myocardial Perfusion Using Nuclear Cardiology Would Enhance Coronary Artery Disease Patient Care-Which Imaging Modality Is Best for Evaluation of Myocardial Ischemia? (SPECT-Side). Circ J 2011; 75: 713-23.

8. Greenwood JP, Maredia N, Younger JF, Brown JM, Nixon J, Everett CC, et al. Cardiovascular magnetic resonance and single-photon emission computed tomography for diagnosis of coronary heart disease (CE-MARC): a prospective trial. Lancet 2012; 379: 453-60.

9. Schwitter Juerg, Wacker Christian M, Wilke Norbert, AlSaadi Nidal, Sauer Ekkehart, Huettle Kalman, Schonberg Stefan O, Luchner Andreas, Strohm Oliver, Ahlstrom Hakan, Dill Thorsten, Hoebel Nadja, and Simor Tamas, for the MR-IMPACT Investigators MR-IMPACT II: Magnetic Resonance Imaging for Myocardial Perfusion Assessment in Coronary artery disease Trial: perfusioncardiac magnetic resonance vs. single-photon emission computed tomography for the detection of coronary artery disease: a comparative multicentre, multivendor trial. Eur Heart J. 2012 Mar 4. [Epub ahead of print].
10. DePasquale EE, Nody AC, DePuey EG, García EV, Pilcher G, Bredlau C, et al. “Quantitative Rotational Thallium-201 Tomography for identifying and Localizing Coronary Artery Disease". Circulation 1988; 77: 316-27.

11. Dunn RF, Freedman B, Bailey IK, Uren RF, Kelly DT. Exercise thallium imaging: location of perfusion abnormalities in single-vessel coronary disease. J Nucl Med 1980; 21: 717-22.

12. Svane B, Bone D, Holmgren A. Coronary angiography and thallium-201 single photon emission computed tomography in single vessel coronary artery disease. Acta Radiol 1990; 31 (3): 237-44.

13. Elhendy A, van Domburg RT, Bax JJ, Poldermans D, Sozzi FB, Roelandt JR. Accuracy of dobutamine technetium $99 \mathrm{mTc}$ sestamibi SPECT imaging for the diagnosis of single-vessel coronary artery disease: comparison with echocardiography. Am Heart J 2000; 139 (2): 22430.

14. Kamihata H, Kurimoto T, Shimada T, Karakawa M, Matsuura T, Ogura E, et al. Infarct size related to the distribution and site of coronary artery lesions studied by the unfolded map technique using single photon emission computed tomography. J Cardiol 1988; 18 (1): 31-41.

15. Pichard AD, Wiener I, Martínez E, Horowitz S, Patterson $\mathrm{R}$, Meller J, et al. Septal myocardial perfusion imaging with thallium-201 in the diagnosis of proximal left anterior descending coronary artery disease. Am Heart J 1981; 102: 30-6.

16. Yoon J-K, Lee K-H, Lee EJ, Kim YH, Seo J-D, Kim B-T. Identification of Proximal Left Anterior Descending Artery Stenosis With Thallium-201 defect Patterns in Patients with Angina Pectoris. Am J Cardiol 2004; 93: 549-53.

17. Verbernea HJ, Fleuryb E, Righettib A, Somsenc GA. The use of perfusion scintigraphy to differentiate proximal right coronary artery stenosis from distal right coronary artery stenosis in patients with inferior left ventricular ischaemia. Nucl Med Commun 2007; 28: 35-40.

18. Slomka PJ, Berman DS, Xu Y, Kavanagh P, Hayes SW, Dorbala S, et al. Fully automated wall motion and thickening scoring system for myocardial perfusion SPECT: Method development and validation in large population. J Nucl Cardiol 2012; 19 (2): 291-302. Epub 2012 Jan 26.

19. Sharir T, Germano G, Waechter PB, Kavanagh PB, Areeda JS, Gerlach J, et al. A New Algorithm for the Quantitation of Myocardial Perfusion SPECT. II: Validation and Diagnostic Yield. J Nucl Med 2000; 41: 720-7. 